\title{
Les débuts de la réglementation française de radioprotection
}

\author{
J. HÉBERT ${ }^{1}$
}

(Manuscrit reçu le 17 décembre 2004, accepté le 11 avril 2005)

RÉSUMÉ La France a réglementé la radioprotection des travailleurs dès 1934. Mais, face à un risque nouveau et sans doute une certaine incertitude sur les moyens de le prévenir, c'est une réglementation qui fut provisoirement adoptée et de surcroît peu contraignante à l'égard des industriels du radium ou des services de radiologie $\mathbf{X}$. Cette réglementation, prise sur le fondement du code du Travail, est demeurée formellement en vigueur jusqu'en 1966. Néanmoins pour les établissements placés sous l'autorité du ministre de la Santé, des circulaires avaient progressivement renforcé le caractère contraignant de certaines dispositions jusqu'à l'intervention en 1966 et 1967 de deux décrets sur la radioprotection des populations et des travailleurs.

ABSTRACT The beginning of French regulation about radiation protection

France has regulated early the protection of workmen against ionizing radiations (1934). But this regulation suffered of inconstancy because the employer's duties were formally limited to deliver to workmen an advice on precautionary measures, when a number of these measures supposed that the employer would provide the means to observe them. Declared to be only provisory, this regulation remained theorically in force till 1966, although replaced in fact by orders of the Health Ministry, at least for hospitals under his authority.

Keywords: French regulation / Occupational radiation protection

\section{Le décret du 5 décembre 1934 : premier texte réglementaire de radioprotection}

Un décret du 5 décembre 1934 et deux arrêtés du 26 décembre 1934 (JO 01/01/1935) ont édicté les «précautions particulières » à prendre pour éviter les dangers que présentent les «corps radioactifs» et les rayons X. Cette réglementation était fondée sur les articles 67 et suivants du code du Travail (selon la rédaction alors en vigueur) et sur le décret du 10 juillet 1913 modifié.

\footnotetext{
${ }^{1} 25$ chemin du trou Martin, 78380 Bougival, France.
} 
Cette réglementation de 1934 bien qu'intervenant près de 40 ans après la découverte des rayons $\mathrm{X}$ par Rœentgen, était cependant antérieure à la plupart des réglementations analogues d'autres pays ${ }^{2}$.

L'attention de l'Administration française avait en effet été attiré dès 1923 sur les dangers que couraient les ouvriers des établissements où l'on se servait de « corps radioactifs » et par l'émotion provoquée par des décès en 1925 et 1926 de chimistes ayant manipulé du thorium ou ayant été exposés aux rayons gamma. L'Académie de médecine demanda en 1929 l'étude d'une réglementation. Une enquête était d'ailleurs en cours depuis 1926 au ministère du Travail, avec la collaboration du professeur Regaud, directeur de l'Institut du radium et du syndicat des ingénieurs chimistes. Cette enquête fut étendue en 1928 aux établissements employant les rayons $\mathrm{X}$. Les résultats de cette double enquête furent soumis en 1928 à la Commission d'hygiène industrielle, qui émit l'avis qu'en raison du petit nombre de travailleurs intéressés il serait préférable de s'en tenir à de simples recommandations, au lieu de prescriptions légalement obligatoires et chargea le professeur Regaud et M. Ferroux, également de l'Institut du radium, de rédiger conjointement des projets de recommandations tant pour la protection contre les « corps radioactifs» que contre les rayons X. Ces projets furent adoptés en 1932 par la commission précitée. Le Comité consultatif des arts et manufactures fut également consulté. Le Conseil d'État marqua sa réticence à l'égard de l'économie du texte en ne lui donnant qu'un caractère provisoire ${ }^{3}$.

Cet historique suggère les observations suivantes : les obligations d'affichage ou de remise d'avis sont de pratique constante dans les décrets relatifs à la protection de la santé des travailleurs. La remise à chaque travailleur d'un avis sur les dangers qu'il pourrait courir et les précautions à prendre, au lieu d'un affichage dans l'atelier, pouvait se justifier en raison du faible nombre de travailleurs employés dans les cinq établissements utilisant alors des « corps radioactifs » ou par la dispersion de ceux qui travaillaient dans les cabinets de radiologie. Un décret du $1^{\text {er }}$ octobre 1913 (JO 12/10/1913) sur l'emploi du ciment à prise rapide constituait un précédent. Mais on ne voit pas en quoi ce faible effectif justifiait le choix de «simples recommandations » contenues dans un simple avis, à la place de prescriptions légalement contraignantes, comme de règle dans un texte réglementaire. Nous sommes réduit à des hypothèses sur les motivations véritables

${ }^{2}$ À l'exception d'un règlement de Basse Autriche du 21 octobre 1889 signalé par B. Moser et de la loi n 147 du 15 avril 1930 du Danemark, il faut attendre, en Allemagne la Rœntgen Verordnung du 7 février 1941 et la Mutterschutzgezetz du 17 mai 1942. Toutefois elles avaient été précédées par des DIN, de même qu'aux États-Unis des limites de doses avaient été fixées, aussi en 1934, par la National Council on Radiation Protection and Measurements. En Grande-Bretagne, il fallut attendre les Factories (luminising) Special Regulations de 1947 et le Radioactive Substances Act de 1948 et en Belgique les arrêtés du Régent des 11 février 1946 et 27 septembre 1947 etc.

${ }^{3}$ A.N. carton AL4122 dossier 211665. 
de la Commission d'hygiène industrielle : esprit de coopération montré par les quelques employeurs utilisant des « corps radioactifs », souci de ne pas heurter le corps médical concerné par un large usage des rayons $\mathrm{X}$ ?

Comme le montre l'exemple allemand des DIN, cela n'aurait été possible que s'il avait existé en France un organisme professionnel, capable d'obtenir de ses membres une observation volontaire d'un « code de bonne conduite».

Nous avons vu par contre que l'Académie de médecine avait demandé une réglementation. Mais dès lors que l'on demandait au ministère du Travail de résoudre le problème de la radioprotection des travailleurs, il ne pouvait le tenter que par le moyen juridique que lui donnait la code du Travail, c'est-à-dire par les dispositions légalement contraignantes d'un décret.

Toutefois en limitant l'obligation de l'employeur à la distribution d'un avis sur les précautions à prendre, le décret de 1934 s'écartait du modèle habituel, car tous les autres décrets de cette nature mettent expressément à la charge de l'employeur des obligations précises comme la remise, le lavage, l'entretien de vêtements de travail ou appareils de protection, la «mise à disposition d'objets nécessaires aux soins de propreté », l'obligation de conclure un contrat avec un médecin pour assurer la contrôle médical, etc.

Le rapporteur au Conseil d'État a évidemment vu ces contradictions et ces insuffisances. Car il écrit « qu'il peut parâtre assez choquant de recommander au personnel ce qu'il appartient au patron de faire ». Au mieux l'avis lui apparaît comme « un mode de pression déguisé » car « la lecture de l'avis par le personnel doit l'amener à constater si le patron a fait ou non ce qui est contenu dans les recommandations ». En conséquence, outre une mention expresse du décret du 10 juillet 1913 rendant applicables des mesures générales, comme d'assurer la ventilation et la propreté des locaux, il suggérait de prévoir expressément, comme mesure particulière, l'établissement d'écrans en plomb. Le Conseil n'a suivi qu'en partie son rapporteur, par la mention du décret de 1913, mais en renvoyant à plus tard le soin de fixer par des règlements d'administration publique (RAP) des «mesures particulière », donnant ainsi un caractère transitoire aux textes de $1934^{4}$.

Le Conseil d'État ne pouvait certes prévoir que le décret, objet de son avis, demeurerait, au moins théoriquement, en vigueur jusqu'au décret $n^{\circ} 66-450$ du

\footnotetext{
${ }^{4}$ Certains décrets de 1913 ou 1935 prévoyaient des mesures transitoires, mais très limitées dans le temps, par exemple sur les délais de mise en œuvre après la publication du nouveau décret et plus généralement les textes réglementaires expressément qualifiés de provisoires sont rares, par exemple les textes pris pour la durée des hostilités. On notera aussi qu'à l'époque l'absence de mesures contraignantes ne pouvait être supplée par des dispositions prises en vertu de la réglementation des établissements dangereux, incommodes ou insalubres.
} 
20 juin 1966 relatif aux principes généraux de protection contre les rayonnements ionisants et même jusqu'aux décrets n ${ }^{\circ}$ 67-228 du 15 mars 1967 et $\mathrm{n}^{\circ}$ 75-306 du 28 avril 1975 relatifs à la protection des travailleurs.

Le texte des avis prévus par le décret de 1934 précité a été fixé par arrêtés du 26 décembre 1934 (JO 01/01/1935).

\section{Avis concernant les dangers présentés par les corps radioactifs}

L'avis comporte deux paragraphes. Le premier est un exposé des dangers imputables à l'action des corps radioactifs et des principes généraux de prévention. Bref, il s'agit d'un résumé de cours sur l'action des rayonnements ionisants, qui n'apportera rien d'autre aux lecteurs qu'un état des connaissances à sa date. Il distingue les accidents cutanés et les accidents intéressant les tissus formateurs des globules du sang, en indiquant en note que des accidents sur les testicules et les ovaires ont également été signalés ${ }^{5}$. S'agissant des accidents sur les tissus formateurs du sang, l'avis distingue l'action des rayonnements selon qu'ils proviennent de sources extérieures ou intérieures à l'organisme. Curieusement ces deux paragraphes contiennent déjà des prescriptions de précautions. À l'égard des accidents cutanés, il indique qu'il ne faut jamais toucher avec les doigts les corps radioactifs et préconise l'emploi d'instruments spéciaux évitant le contact de l'épiderme et assurant un éloignement indispensable des émetteurs bêta et gamma. À l'égard des accidents intéressant les tissus formateurs des globules sanguins, créés par des sources extérieures de rayonnements, il indique que l'on s'en préserve par l'éloignement ou l'interposition d'écrans. Pour les sources intérieures, principalement les "dépôts actifs », il faut faire en sorte que les récipients contenant des matières actives soient toujours séparées de l'atmosphère générale des locaux où sont les travailleurs et assurer une bonne ventilation de ces locaux. Il met spécialement en garde contre les «pratiques fort imprudentes » qui consistent à utiliser pour boire des «récipients ayant contenu des corps radioactifs ».

Bien que la seule obligation expresse des employeurs posée par le décret se réduisît à la distribution de l'avis, l'avis " appelle instamment l'attention des employeurs comme du personnel» sur les «recommandations essentielles » développées dans la seconde partie, où si certaines précautions doivent en effet être prises par les travailleurs eux-mêmes, ceux-ci ne peuvent les respecter que si les employeurs ont de leur côté réalisé des aménagements ou mis à disposition de leur personnel les moyens nécessaires. Malgré l'ambiguïté persistante, il semble que

${ }^{5}$ Mais leur attribue un caractère de gravité moindre, tout en les assimilant pour les moyens de protection à mettre en œuvre aux atteintes aux tissus sanguino-formateurs. 
l'inspection du travail pouvait cependant utiliser la procédure de mise en demeure, prévue par le code du Travail dans ce domaine de la protection de l'hygiène et de la sécurité. De fait les «bulletins du ministère du Travail » citent deux « mises en demeure » en 1935 et 1937 fondées sur le décret de 1934, mais sur un total de 4449 et 2239 et sans préciser l'objet de la mise en demeure, qui pourrait avoir été les prescriptions de portée générale du décret de 1913. Cette statistique n'a plus été publiée après 1939. Mais ce serait sans doute de l'angélisme de croire à une meilleure application du texte après 1939 et au mieux on peut espérer que le décret de 1934 a pu contribuer à la prise de quelques bonnes habitudes.

La seconde partie de l'avis développe les mesures préventives à appliquer pour cinq catégories d'activités industrielles et marque la nécessité du contrôle de l'efficacité des mesures de précautions prise et de la surveillance médicale du personnel.

Quatre catégories sous entendent que l'industrie du radium est concernée.

\subsection{Conservation, manutention, manipulation, triage, broyage et traitement mécanique des minerais}

Les seules précautions que doivent prendre les salariés sont de ne pas séjourner sans nécessité dans les locaux consacrés à ces opérations, d'y revêtir des effets spéciaux remplaçant ou recouvrant leurs effets ordinaires, et de procéder à la fin de la période de travail à une toilette soigneuse des parties découvertes du corps, précédée par un dépoussiérage complet des effets et des chaussures.

Mais il est clair que seuls les employeurs pouvaient isoler les locaux, les éloigner autant que possible des autres lieux fréquentés par le personnel, fournir, garder à l'usine et entretenir les « effets spéciaux », procurer les aspirateurs, etc.

\subsection{Gros traitements chimiques de minerais (attaque, lessivage, décantation)}

Après avoir énuméré les diverses destinations des salles ou laboratoires où pouvaient s'effectuer ces opérations, cet alinéa distingue la protection contre le rayonnement direct et les précautions à prendre contre les poussières et gaz actifs

Dans l'un et l'autre cas, les prescriptions explicites faites aux travailleurs ne consistaient qu'en l'usage des instruments, ou gants en caoutchouc, appropriés aux manipulations, dans la toilette soignée à la fin de la période de travail et de n'abandonner « ne fût-ce que quelques instants... des foyers radioactifs... sans les entourer d'un écran de plomb épais ». 
Mais seul l'employeur était en mesure d'agencer les locaux de telle sorte que les travailleurs ne restent au voisinage des matières actives que lorsque cela est indispensable pour le travail et en particulier de disposer convenablement les machines et récipients et de placer les dispositifs de protection par écrans ou blindages, notamment de disposer de tables comportant une planche de plomb et des écrans verticaux. S'agissant plus particulièrement de la protection contre les poussières et les émanations gazeuses, les corps radioactifs ne doivent jamais êtres abandonnés à découvert dans des locaux où séjourne le personnel et il est dit que l'élimination des émanations gazeuses (radon, thoron) doit être assuré très soigneusement, par réalisation des opérations sous des cônes d'aspirations de hauteur réglable ou, de préférence, dans des hottes fermées, dont l'atmosphère sera expulsée directement à l'extérieur, par des conduits munis de ventilateurs. Les opérations étant surveillées à travers des glaces en verre plombé, tandis qu'une bonne ventilation des locaux permettra aux travailleurs de respirer un air exempt de toute matière active.

\subsection{Conservation des produits concentrés}

Les prescriptions concernant les meubles et les locaux où sont conservés des corps radioactifs concentrés ne peuvent être suivies que par les employeurs, car elles consistent dans l'éloignement de ces meubles et locaux de tout local où séjourne habituellement le personnel et dans la mise en place de blindage de plomb dans leurs parois.

Il convient de noter que les épaisseurs de plomb préconisées en note reprennent une des recommandations formulées par la Commission internationale de protection contre les rayons $\mathrm{X}$ et les rayons du radium (aujourd'hui la CIPR) au cours du $3^{\mathrm{e}}$ Congrès international de radiologie tenu à Paris en juillet 1931.

\subsection{Transport des produits purs concentrés}

Il ne doit être pratiqué l'intérieur de l'usine ou laboratoire que par le moyen de récipients à parois garnies de plomb. Là aussi seul l'employeur est à même de mettre à disposition les boîtes, coffrets, etc. nécessaires, voire même un moyen de transport évitant le portage. La seule obligation implicite du travailleur est d'utiliser les moyens mis à sa disposition.

\subsection{Produits radioactifs lumineux}

Avec eux on sort de l'industrie du radium pour s'intéresser à la radioprotection dans l'industrie horlogère, qui avait découvert et développé l'usage des peintures luminescentes. L'exposé est assez contradictoire. En effet, après avoir affirmé que 
ces produits sont en général « forts peu actifs » et que certains n'émettent que du rayonnement alpha et que par suite leur manipulation « est exempte de danger », il déclare qu'il ne faudrait cependant pas les considérer « comme incapables de causer des accidents », en signalant les nécroses de la muqueuse buccale et des maxillaires, ou les anémies pernicieuses et tumeurs « la plupart du temps mortels » signalées à l'étranger (comme on le sait aux États-Unis), chez les ouvriers des deux sexes «qui avaient pris la déplorable habitude d'appointer leurs pinceaux avec leurs lèvres ». Mais la précaution consistant à s'abstenir d'une semblable pratique n'est qu'implicite.

S'agissant des mesures de contrôle pour s'assurer de l'efficacité des précautions prises, celles-ci devaient consister en des mesures directes de rayonnement dans les locaux où séjourne le personnel et dans des prélèvements de l'air respiré par le personnel pour y pratiquer un dosage de radioactivité. Là aussi seuls les employeurs étaient en mesure de pratiquer ces contrôles.

En ce qui concerne la surveillance médicale du personnel, une surveillance attentive devait être «nécessairement » complétée par l'examen microscopique du sang, pratiqué tous les six mois au moins, par un médecin «spécialement compétent, toujours le même», qui consignera ses observations et résultats d'analyse sur un registre où chaque travailleur aura sa place réservée.

La seule obligation, ici aussi implicite des travailleurs, était de se soumettre à cette surveillance et aux prélèvements de sang. Les employeurs doivent prendre les mesures d'organisation du travail nécessaire et conclure un contrat avec un médecin compétent. Sur ce point, l'avis est plus précis que la recommandation de la Commission internationale, adoptée en 1931, par amendement à celle de 1928, qui ne prévoyait pas cette surveillance médicale.

L'avis se termine curieusement par un nota, indiquant que pour suppléer à l'insuffisance possible de ces recommandations (une modestie rarement rencontrée dans la réglementation française !) ou en présence de cas particuliers imprévus, il était recommandé aux chefs d'établissement de faire appel au concours d'un « organisme scientifique compétent» qui, après visite des lieux, donnera tous avis ou conseils utiles.

\subsection{Avis concernant les rayons $X$}

De structure analogue à celle de l'avis précédemment examiné, il s'en distingue non seulement par les conséquences des différences existant entre les sources d'irradiation, mais aussi en faisant sans le dire la leçon aux radiologues euxmêmes. Car à la différence des chefs d'établissement du cycle du radium, ceux-ci manipulent eux-mêmes leurs appareils et devraient donner l'exemple. 
Le premier paragraphe, comme dans l'avis précédent, est consacré aux dangers inhérents à l'action des rayons X, et distingue les accidents cutanés de ceux qui intéressent les tissus formateurs des globules du sang. Les alinéas consacrés aux accidents cutanés sont plus développés que dans l'avis précèdent et dénoncent dans l'introduction «consciente» directe des mains dans le champ de rayonnement « une imprudence ou une insouciance tout à fait inadmissible » et insistent aussi sur le risque crée par le cumul d'exposition par « inadvertance, par habitude, par insouciance ou par curiosité ». S'agissant des accidents intéressant les tissus formateurs du sang, cet avis attire l'attention sur des écrans insuffisamment opaques, la diffusion dans d'autres directions que celle du faisceau, ou par les murs, l'air, etc.

Les mesures préventives, sur lesquelles l'attention des employeurs et du personnel est «instamment» appelée, consistent dans le placement des tubes radiogènes dans une enceinte close et dont les parois ont été rendues opaque aux rayons $\mathrm{X}$ par une épaisseur de plomb préconisée, en fonction de la tension électrique, par la Commission internationale de protection contre les rayons $\mathrm{X}$ et les rayons du radium lors de son $3^{\mathrm{e}}$ congrès de Paris en 1931.

Cette référence à des recommandations d'une ONG parait être à l'époque une innovation. Toutefois, on en aura noté la portée limitée à une disposition de caractère purement technique et il est à noter en particulier que le décret n'a pas repris (et ne pouvait pas d'ailleurs le faire) les restrictions du temps de travail (40 heures par semaine, 4 semaines de congé consécutives) préconisées par la Commission. On sait qu'il a fallu attendre 1936 pour que les 40 heures deviennent la durée légale, plus 15 jours de congés annuels ! Toutefois en 1928 et 1931 la Commission ne se préoccupait pas des patients, mais seulement des opérateurs et se bornait à poser qu'un opérateur ne devait en aucun cas s'exposer «sans nécessité » au faisceau direct de rayons $\mathrm{X}$.

Aucune référence au principe de justification n'est non plus faite, alors qu'il avait déjà été parfaitement posé par la jurisprudence française à propos de l'épilation par rayons X. Nous sommes donc ici en présence de développements indépendants et dans le cas de cette jurisprudence sans influence sur l'élaboration ultérieure du principe par la $\mathrm{CIPR}^{6}$.

\footnotetext{
${ }^{6}$ Paris 22 janvier 1913 Dr Delherm c/ Dlle Calou DP-1919-2-73, approuvé par Cass. Civ. 24 novembre 1920 Gaz. Pal-1921.28, S-1921-1-11 ; DP-1924-1-104 La cour d'appel «considérant que la radiothérapie peut avoir des conséquences graves... considérant dès lors que le médecin ne doit pas hésiter à appliquer ce traitement lorsque la santé du malade l'exige... il n'en est pas de même lorsque le médecin se trouve en présence... d'une simple imperfection physique à faire disparaître ou dissimuler... que la Dlle N n'avait qu'un peu de barbe au menton, que sans doute sa coquetterie en souffrait, mais que le Dr D n'allègue même pas qu'il y eût... une obsession quasi maladive...; il avait le devoir de refuser son concours et qu'il n'établit même pas qu'il ait prévenu cette jeune fille du danger qu'elle pouvait courir, sa faute est manifeste... le préjudice n'en est pas moins certain, mai qu'il ne faut pas en exagérer l'importance... que les conséquences actuelles se réduisent à des lésions de la peau du menton...; indélébiles... pas plus disgracieuses... que des poils nombreux et développés qu'elle portait au menton avant le traitement ». Le Dr Jammet avait connaissance d'un cas de cancer attribuable à un tel traitement épilatoire. Nous avions comparé nos données pour conclure qu'il s'agissait de deux personnes différentes.
} 
L'efficacité des dispositifs de protection doit être vérifié par des mesures fluoroscopiques, radiographiques, et surtout électroscopiques, à la fois dans les locaux où le personnel est appelé à séjourner au voisinage de tubes en activité, mais aussi dans les salles voisines. Toutefois aucune disposition ne règle la fréquence de ces contrôles.

Une surveillance médicale du personnel doit être effectué dans les mêmes conditions que dans l'industrie du radium.

\section{La période de la radioprotection par voie de circulaire}

\subsection{Circulaire du 17 juin 1937}

Elle concerne la protection du personnel dans les établissements de soins contre les rayonnements nocifs des appareils de radiologie et de curiethérapie (JO 19/06/1937). Cette circulaire, qui ne fait curieusement pas référence aux textes de 1934, s'applique aux hôpitaux ou dispensaires placés sous tutelle du ministre de la Santé publique, et apporte les compléments et précisions suivantes.

Dans les laboratoires de recherches ou de traitement, il devra être procédé au moins deux fois l'an par les «personnes qualifiées »

1. à des mesures directes de rayonnements et à un dosage de radioactivité dans les locaux où séjourne le personnel,

2. à des prélèvements de l'air respiré par le personnel et à un dosage de radioactivité.

Dans tous les hôpitaux ou dispensaires où se pratiquent les examens radiologiques (rappelons que nous sommes à l'époque en pleine lutte contre la tuberculose) le personnel, appelé soit à manipuler les appareils soit à approcher les malades lors de ces examens, doit faire l'objet d'une analyse semestrielle microscopique du sang «par un médecin spécialement compétent en hématologie » et «à même de suivre le personnel au cours de plusieurs examens ». Ce praticien devait consigner ses observations sur un registre où chaque personne soumise à la surveillance médicale devait avoir une place réservée. L'inspecteur départemental d'hygiène devait viser ce registre et procéder à une enquête, en cas d'altération du sang, sur les mesures d'éviction du personnel malade et les modifications à apporter aux installations.

Le ministre ajoutait qu'il attachait la plus grande importance à ce que toutes les installations de diagnostics ou de thérapeutique soient munies des dispositifs de protection nécessaires pour éviter l'action nocive des rayonnements directs ou 
secondaires et qu'il tiendrait compte de la manière dont ces prescriptions auront été observées dans la distribution des subventions. Les inspecteurs devaient aussi rendre compte de chaque cas de début d'anémie pernicieuse. L'attention du ministère avait en effet été attirée auparavant sur l'altération de l'état de santé du personnel des établissements de cure. Il est à souhaiter qu'un jour les archives soient ouvertes sur ce sujet, si elles existent encore.

\subsection{Circulaire $n^{\circ} 144$ du 6 novembre 1941 sur la protection du personnel hospitalier contre les corps radioactifs ${ }^{7}$}

Cette circulaire débute ainsi : «Il a été porté à ma connaissance que, dans la plupart des établissements hospitaliers et sanatoriums, les mesures de protection prescrites par les arrêtés de M. le ministre du Travail en date du 26 décembre 1934 en faveur du personnel manipulant les appareils de radiologie et les corps radioactifs ne sont presque jamais appliqués. Il en résulte que des accidents, parfois très sérieux, se produisent assez souvent parmi le personnel de ces établissements ».

La circulaire renvoie aux prescriptions de 1934, en insistant sur la surveillance médicale comportant un examen radioscopique semestriel du sang par un médecin spécialiste.

Bien que ce texte ait insisté auprès des directeurs d'hôpitaux sur la nécessité d'appliquer les prescriptions de 1934 et de mettre en pratique les mesures de protection envisagées, nous savons, par le témoignage de M. le professeur Tubiana, que jusque dans les années 50 la radioprotection laissait encore à désirer même dans un hôpital réputé.

On aura noté aussi l'insistance mise sur les examens du sang au cours de cette période. Or si ces examens peuvent attirer l'attention sur des défaillances de la protection, ils ne procurent pas celle-ci.

\section{L'après-guerre}

Toutefois en ces années de guerre et de l'après-guerre, la préoccupation principale des autorités a été de procurer aux établissements hospitaliers et autres les moyens de travailler et nous citerons à titre d'exemple :

- la circulaire Santé du 4 novembre 1940 prescrivant le recensement du radium existant en France ${ }^{8}$,

\footnotetext{
${ }^{7}$ Bulletin du secrétariat d'État de la famille et de la santé, textes officiels concernant la protection de la santé et de la famille publiés en 1941, Imprimerie nationale 1941, p. 130

${ }^{8}$ Ministère de la Santé, Table générale de législation sanitaire française 1780-1955, Vol. II, Imprimerie nationale 1955.
} 
- l'ordonnance n $45-2722$ du 2 novembre 1945 sur l'acquisition par les hôpitaux et établissements d'assistance de matériels hospitaliers d'origine américaine (JO 05/11/1945) et la circulaire $\mathrm{n}^{\circ} 224$ du 31 août 1945 sur la répartition de ces matériels (Table précitée, année 1945, p. 59),

- la circulaire $\mathrm{n}^{\circ} 182$ du 10 juillet 1947 relative à l'approvisionnement en films radiologiques (idem., p. 473),

- l'arrêté du 6 décembre 1949 portant application de l'article 2 du décret du 30 juillet 1949 créant la Commission interministérielle pour l'achat de radioéléments à l'étranger (JO 09/12/1949).

Ce n'est qu'au cours des années 50, que, l'étreinte de la pénurie se desserrant, la préoccupation de la radioprotection a pu réapparaitre dans la réglementation, sinon encore dans la pratique. Ce renouveau s'est exprimé par des textes pris dans le cadre de réglementations diverses.

Citons inter alias :

- l'arrêté du 27 juin 1952 (JO 05/07/1952) fixant les règles techniques d'établissement et d'installation des appareils de radiologie,

- l'arrêté du 7 avril 1953 (JO 15/04/1953) fixant la liste des organismes habilités à délivrer des certificats d'étalonnage et de réétalonnage attestant du bon fonctionnement des dosimètres et appareils de mesure,

- des décrets n ${ }^{\circ} 53-1001$ du 5 octobre 1953 (JO 07/10/1953) et 54-475 du 3 mai 1954 (JO 07/05/1954) et $\mathrm{n}^{\circ}$ 55-512 du 11 mai 1955 (JO 12/05/1955) modifiant le code de la Santé publique en créant la CIREA et organisant le régime des radio-éléments artificiels.

Mais au cours de la seconde moitié des années 50 s'ouvre un nouveau chapitre de l'histoire de la réglementation de la radioprotection, avec la négociation du traité de Rome EURATOM du 25 mars 1957 et de son chapitre sur la «protection sanitaire », suivi plus tard le 2 février 1959 par les «normes de base » et leur mise en application par circulaire du 7 mars 1962. Toutefois cette circulaire ne constituait qu'un bricolage, le principe de hiérarchie des textes ne lui permettant évidemment pas d'abroger le décret de 1934. La création du SCPRI par arrêté du 13 novembre 1956 (JO 15/11/1956) a également été un fait marquant.

\section{Conclusion}

Cette première réglementation souffrait d'un défaut originel. Le souhait de n'émettre que de «simples recommandations» était en contradiction avec l'adoption d'un instrument réglementaire. Cette réglementation était également déséquilibrée. L'obligation de l'employeur se réduisant à la remise d'un « avis » sur les précautions à observer, alors que la plupart de celles-ci supposait une action 
positive de l'employeur. Tombés en désuétude dans la pratique, car remplacé par des circulaires, tout au moins dans le monde hospitalier, puis dans les années 50 et 60 par des réglementations intervenues en ordre dispersé, ces textes de 1934 sont cependant demeurés théoriquement en vigueur jusqu'à la mise en pratique des normes de base d'EURATOM, elles-mêmes fondée sur la doctrine élaborée par la CIPR de relation linéaire sans seuil et d'optimisation.

Citons également l'arrêté du 14 juin 1955 (JO 24/06/1955) instituant auprès du ministre de la Santé publique et de la population une commission chargée d'étudier les problèmes de protection contre les rayonnements ionisants. Nous ignorons quel fut son rôle dans le développement de la réglementation. Un arrêté du 5 mars 1923 (JO 05/05/1923, p. 4471) avait déjà constitué une commission à l'effet d'étudier l'influence éventuelle de l'emploi des rayons X sur le voisinage, ce qui témoignait d'un intérêt précoce pour l'environnement.

Citons également : l'arrêté du 27 juin 1952 (JO 27/06/1952) définissant les règles d'établissement... des appareils de radiologie à l'usage des collectivités publiques... ; l'arrêté du 29 juin 1960 fixant les mesures de... sécurité à prendre par les administrations hospitalières en vue de la protection médicale de leur personnel (JO 07/07/1960) et imposant notamment le port d'un film dosimètre et une dose maximale admissible de 3 rem sur 13 semaines ; la circulaire du 23 février 1957 relative à la protection contre les radiations ionisantes ; le bulletin du ministère de la Santé $n^{\circ} 8 / 57$ et circulaire du 3 juin 1957 relative aux recommandations générales visant la protection contre les radiations ionisantes (JO 11/07/1957)... ; les réglementations internationales ou nationales du transport ont également joué un rôle dans la protection des travailleurs concernés.

Pour le ministère du travail signalons : un arrêté du 13 octobre 1954 (JO 21/10/1954) a également inscrit sur la liste des travaux soumis à surveillance médicale spéciale les travaux comportant la préparation, l'emploi, la manutention ou l'exposition aux rayons $\mathrm{X}$ et substances radioactives et le décret $\mathrm{n}^{\circ}$ 58-628 (JO 24/07/1958) interdisant d'employer des « enfants âgés de moins de 18 ans » ou de les admettre de manière habituelle dans les locaux affectés à des travaux exposant à la radioactivité.

\section{POUR EN SAVOIR PLUS}

Bines W., Chandler S.D. (2000) The development of occupational public and environnemental radiation protection legislation in Great Britain-Proceedings of the IRPA 10 International Congress, Hiroshima, May 2000, pp. 9-124.

Bischof W. (1973) Zur Geschichte des deutschen Strahlenschutzrecht-Fortschrifte Röntgenstrahlen, Kommentar zum Bonner Grundgesetz, Vol. 4, pp. 31-38.

Bischof W., Pelzer N. (1983) Das Strahlenschutz in der Mitgliedstaaten der Europäischen Gemeinschaft, Band II, Baden-Baden. 


\section{LES DÉBUTS DE LA RÉGLEMENTATION FRANÇAISE DE RADIOPROTECTION}

Bulletin du ministère de la Santé publique, Textes officiels concernant la santé publique. Imprimerie nationale (1936)

Recueil des textes officiels concernant la protection de la santé publique 1790-1955. Imprimerie nationale (1957)

Bulletin du Ministère du travail (1934) Berger Levrault éditeur.

Goettinger Atomrecht Katalog, Institut für Volkerrecht der Georg-August Universität Goettingen RFA.

Hébert J. (1999) La jurisprudence française en matière médicale face aux rayonnements ionisants in OCDE-AEN, Droit nucléaire, Bulletin n 63/juin 1999.

Moser Bertold (1990) Die erste Strahlenschutz regelung-Atomwirtchaft, 1990-470.

Pallardy G., Pallardy M.J. (1987) La photographie à travers les corps opaques ou les premiers pas de la radiologie, Bulletin d'histoire de l'électricité, n 9/juin 1987.

Pasquier J.L. (2004) Un siècle d'exposition, quatre-vingt ans de radioprotection, Contrôle n ${ }^{\circ} 158$.

Penneau A. (1989) Règles de droit et normes techniques, LGDJ.

Persson L. (1987) Radiation Protection and Atomic Energy legislation in the Nordic countries Statensstralsinstitut, Stockholm 1987, SSI-Rapport 87-3.

Pinell P. (1992) Naissance d'un fléau, Histoire de la lutte contre le cancer en France, p. 54. Métaillé éditeur, Paris.

Ratkoczy N. (1971) Geschichtliches ûber Strahlenschädigung und Strahlenschutz, Strahlentherapie 141, 311-437.

Reid R. (1979) Marie Curie, derrière la légende. Seuil, Paris. 